\title{
A high precision aeromagnetic survey near the Glen Hummel Field in Texas; Identification of cultural and sedimentary anomaly sources
}

\author{
Clark R. Wilson, GeOrgios TSOFLIAS, and MoniKa BARTELMANN, The University of Texas at Austin, Texas \\ JOE PHILLIPS, World Geoscience, Houston, Texas
}

\begin{abstract}
We present a case study of a high resolution aeromagnetic survey near the Glen Hummel oil field (Figure 1). The survey was originally flown to support a study of aeromagnetic methods for environmental hazard detection, but it provides an opportunity to investigate various problems that arise when ana-
\end{abstract}

lyzing low level total field magnetic anomalies in an area of petroleum production. There is a fair amount of interest in high resolution magnetics as a prospecting tool, given the high data quality now available, and the existence of mechanisms (bacterial and chemical) that may produce anomalies related to petro-

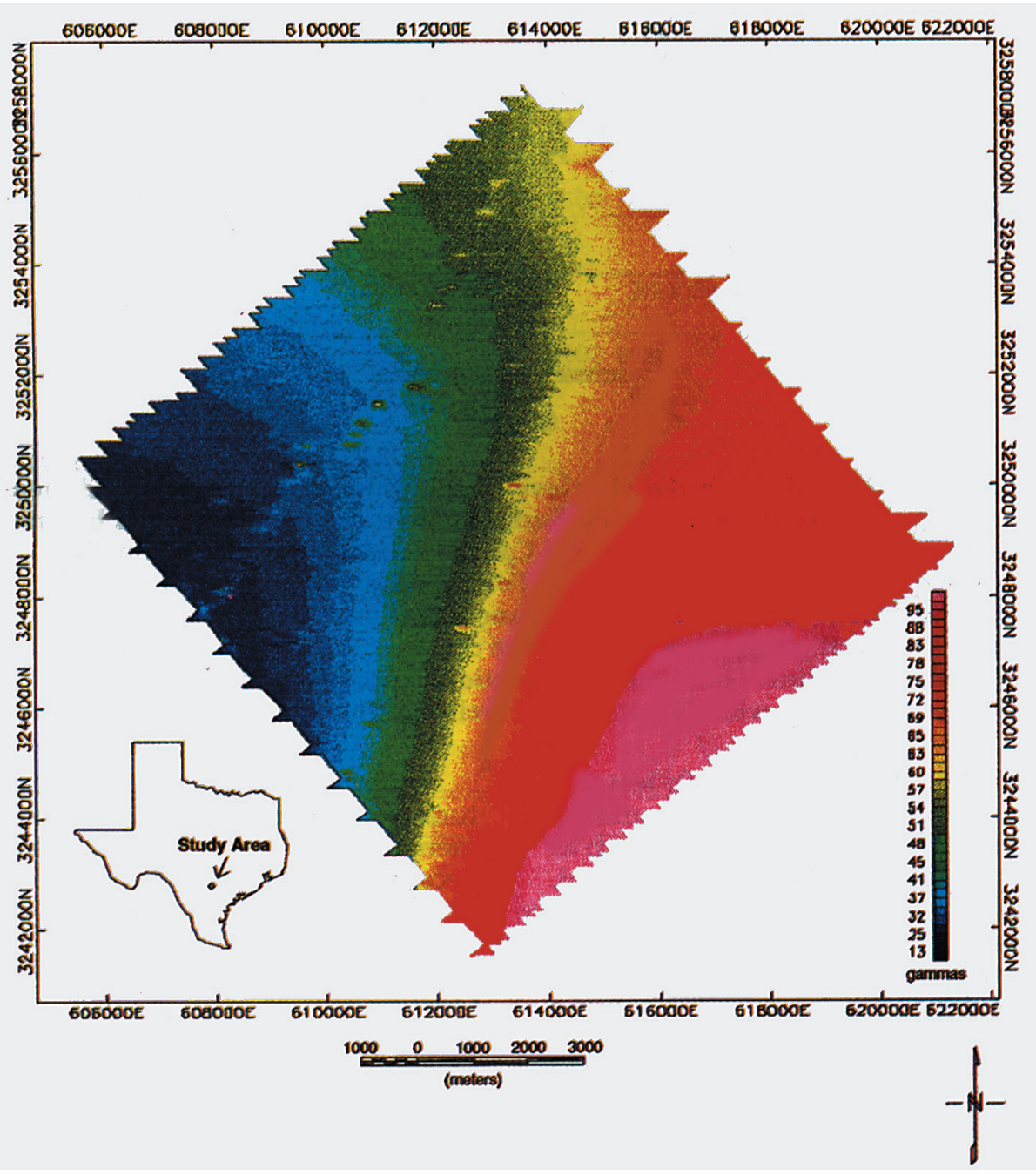

Figure 1. Color contoured map of total magnetic field anomalies in the study area. Dominant regional anomalies with spatial scales of several kilometers are almost certainly due to metamorphic basement sources. Isolated small scale features are due to cultural sources which are revealed more clearly in Figure 2 after subtracting a smooth 3-D order polynomial surface. Inset map shows the location of the survey. Map projection UTM Zone 14 North. leum occurrence. Sedimentary sources of magnetic anomalies near the Glen Hummel Field appear to be original depositional variations in magnetic mineral concentration unrelated to the presence of petroleum. However, the Glen Hummel survey provides a useful illustration of challenges in the interpretation of high resolution aeromagnetic data, especially the problem of separating low level natural source magnetic anomalies from manmade "cultural" anomalies inevitably associated with petroleum production.

The Gulf Coast sedimentary section in this area consists of 3-4 km of sediments, which dip down towards the Gulf, and are underlain by metamorphic basement. Principal sedimentary units of interest are the Wilcox, in which Glen Hummel reservoirs are located, the overlying Carrizo Sand and the Reklaw Formation, the surface unit at Glen Hummel. Fault-bounded reservoirs at depths of $700-800 \mathrm{~m}$ have produced over 3 million bls oil since the 1960s. In addition to oil and gas production, this region of Texas is also known for commercial uranium deposits hosted by sandstones of Eocene and younger ages. The nature of these shallow mineralized zones is of particular interest because many contain magnetic minerals. For example, a deposit in the Miocene Oakville Sand shows evidence of depositional magnetite, some early diagenesis to hematite, and later stage uranium mineralization resulting from fluid migration.

Data acquisition and processing. The aeromagnetic data were acquired in March 1994 by BGM Airborne Surveys in Wilson and Karnes counties, Texas using a stinger-mounted, optically pumped Texas Instruments helium vapor magnetometer. Aircraft positioning was by differential GPS navigation, 
a barometric altimeter, a radar altimeter, and a continuous videotape of ground location taken during the survey. Navigation precision is estimated to be about $2 \mathrm{~m}$.

Data were acquired over a set of $50 \mathrm{~km}$ northwest-southeast flight lines spaced about $400 \mathrm{~m}$ apart, at about $100 \mathrm{~m}$ elevation, draped over the surface. Cross track tie lines, $80 \mathrm{~km}$ long, were spaced $800 \mathrm{~m}$ apart. Geomagnetic data were recorded in the aircraft at a rate of $432 \mathrm{~Hz}$, and were digitally compensated for aircraft motion using two separate 3-axis fluxgate magnetometers. The motion-compensated magnetic total field values were smoothed and resampled at a rate of $10 \mathrm{~Hz}$ for final processing, corresponding to along-track sample spacing of approximately $4 \mathrm{~m}$. Temporal field variations were recorded by a base station instrument at Stinson Airport. Diurnal and other temporal variations determined from the base station were subtracted from the data, and a time delay (parallax correction) associated with the recording system was removed. The IGRF 1990.0 field was removed, and a cross-track datum "leveling" algorithm was applied to adjust for temporal magnetic variations not accounted for by the base-station instrument. The leveling software, a proprietary product of BGM, adjusts only the main flight lines, and the cross-track data are not used after leveling.

After these steps, anomalies remain with amplitudes of tens of gammas, and with dominant spatial scales of several km (Figure 1). These features are undoubtedly due to sources in the metamorphic basement, and are not of interest in this study. Subtraction of a third order polynomial surface effectively removes these larger scale features, leaving the residual flight line data as the starting point for further analysis (Figure 2) . From this point, we use flight profile wiggle traces to display the data, rather than contoured maps such as Figure 1, in order to investigate features near the limits of spatial resolution and amplitude detectability.

Identifying natural-source anomalies. Isolated features, barely visible in Figure 1, are prominent in Figure 2 after removing low spatial frequency basement anomalies.

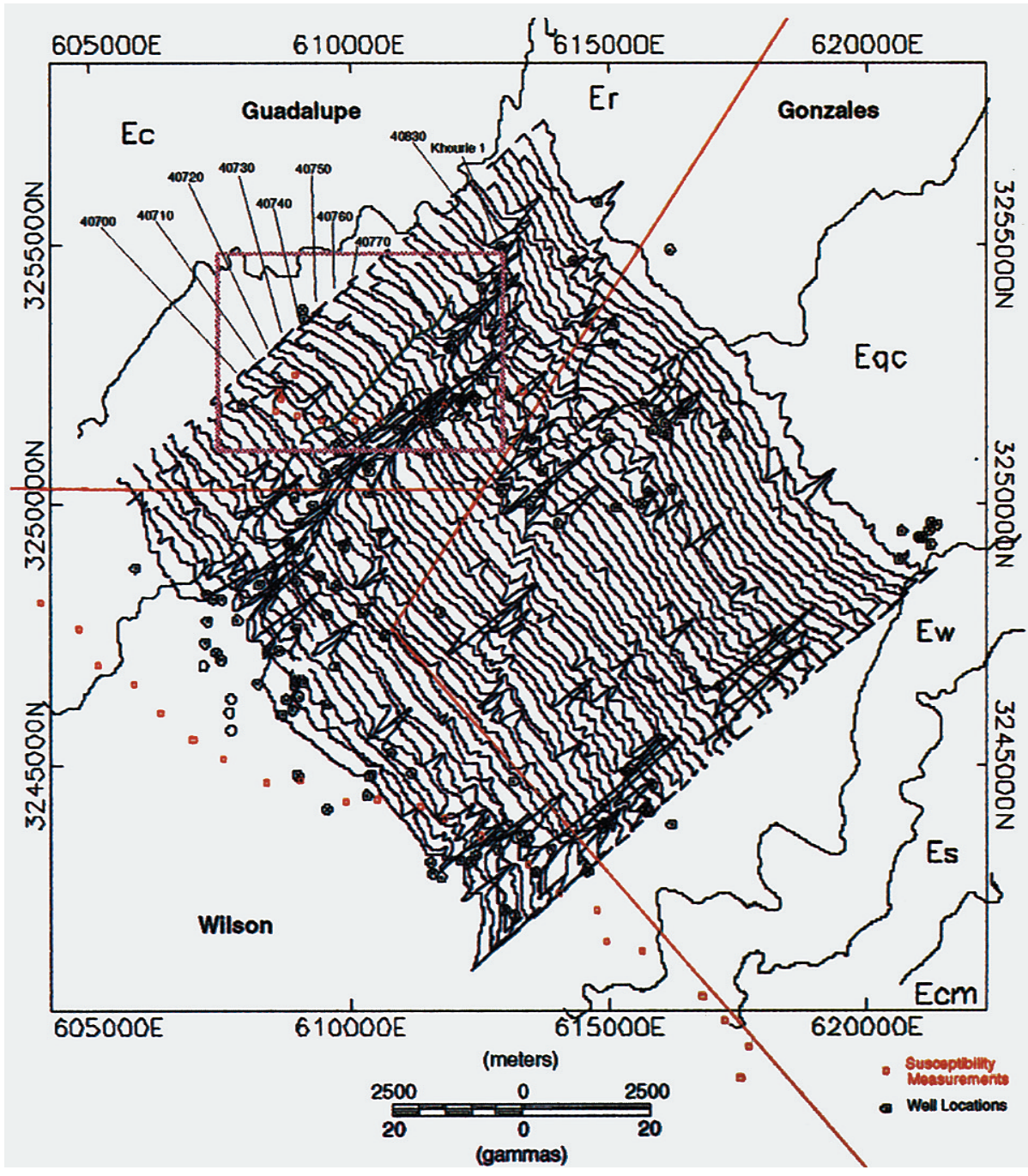

Figure 2. Composite map showing leveled flight line profiles, major sedimentary units, and county boundaries. Surface outcrop nomenclature: $\mathrm{E}=$ Eocene Formation with lower case letters $c=$ Carrizo Sand; $r=$ Recklaw formation; $q c$ = Queen City Formation; $\mathbf{w}=$ Weches Formation; $\mathbf{s}=$ Sparta Sand; and $\mathrm{cm}=$ Cook Mountain Formation. Low level anomalies of a few gammas correlated across several flight lines, roughly in the center of the boxed area, are indicated by the green line. Locations of magnetic susceptibility samples are denoted by red symbols, while identified oil wells are shown with black symbols.

Most of these isolated $\sim 10 \gamma$ features are cultural magnetic anomalies due to man-made structures such as petroleum production equipment, buildings, and casing in abandoned wells. In addition, there are other low level $(\sim 1 \gamma)$ anomalies, which are difficult to see given the plotting scale in Figure 2. Some of these, identified by the green line, are probably of natural origin; this conclusion is based upon cross-line correlations, amplitudes, and the lack of apparent cultural sources. The trend of these low-level anomalies is roughly parallel to major sedimentary unit boundaries and mapped faults, further suggesting a natural geologic source.
Unfortunately, the strongest cultural anomalies also follow sedimentary boundary and fault trends because Glen Hummel reservoirs, hence well locations, tend to be fault-controlled.

Quality control. At this point, it is useful to demonstrate that the data are of sufficient quality to detect very low level anomalies. Although magnetometer sensitivity is certainly adequate, one might wonder whether data processing artifacts dominate at the $\sim 1 \gamma$ level, given that processing has involved adjustments of tens of gammas.

For example, could the leveling algorithm, which adjusts the main 
profiles using the cross-track tie lines, introduce apparent "anomalies" which are correlated across several flight lines? This issue is addressed in Figure 3, which shows a number of flight line profiles from the boxed area of Figure 2 . Unleveled profiles, as recorded in the aircraft, are displayed next to fully processed leveled profiles, with mean values subtracted to facilitate comparison. It is clear that most low amplitude $(\sim \gamma)$ anomalies are preserved in the processing, although, in some lines (e.g. 40730 and 40740) there are features found in the leveled data that are not in the unleveled. These may be artifacts of the data processing. However, there is no clear correlation with the intersecting tie line locations (indicated by $\mathrm{T}$ ). Furthermore, the anomaly pattern that we have identified in Figure 2, and indicated by $\mathrm{A}$ in Figure 3, is present in both leveled and unleveled profiles. Thus, we conclude that data quality is adequate to detect natural-source anomalies below $1 \gamma$.

Cultural anomaly suppression. Using Texas Railroad Commission reports and petroleum industry data bases, we were able to corre-

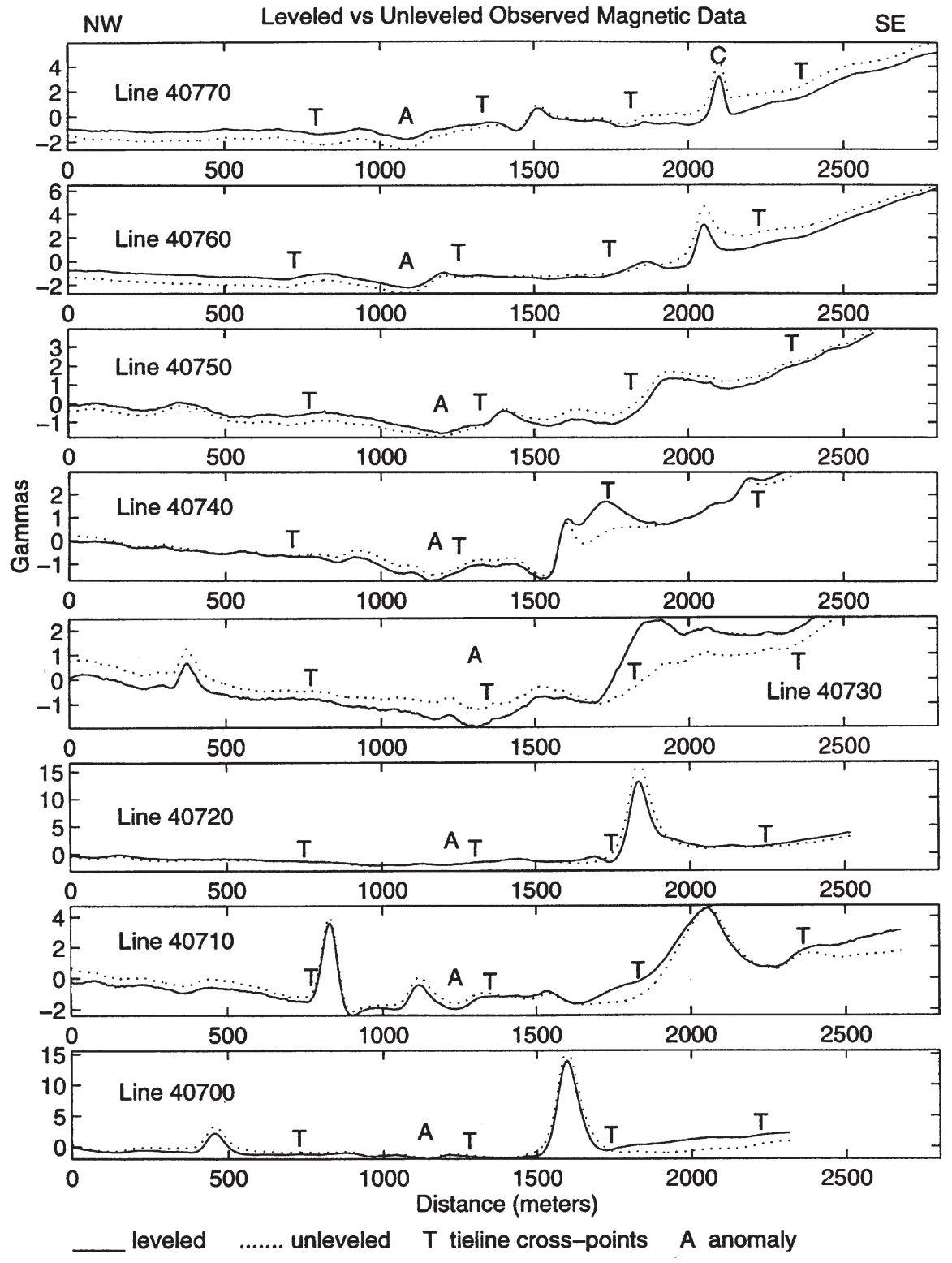

Figure 3. Flight line profiles for several lines in the center of the boxed area of Figure 2. Means have been removed to permit comparison of raw and fully processed data. Note locations of intersecting tie lines indicated by $\mathrm{T}$ and anomalies identified in Figure 2, indicated by $\mathrm{A}$. late many of the isolated magnetic anomalies in Figure 2 with known active or abandoned wells. Thus, aeromagnetic surveying is effective at locating abandoned wells and other environmental hazards when amplitudes are roughly an order of magnitude larger than background levels, as in this case. Conversely, if the background sedimentary magnetic anomalies are the signal, then cultural anomalies are a significant source of noise.

Given their large relative amplitudes, cultural anomalies are difficult to remove by simple linear filtering, because they possess spatial scales similar to near-surface sedimentary anomalies. Cultural anomalies are a nuisance in data display and, perhaps most frustrating, in areas of petroleum production they will probably follow the trends of major geologic boundaries, faults, and structures.

Figure 4 shows a cultural anomaly due to a known well (Khourie 1, drilled in 1985), selected from line 40830 (Figure 2). We computed a simple dipole approximation to the observed anomaly using reported location and other data, as shown in Figure 4. Most identifiable cultural anomalies in Figure 2 have a similar dipole appearance, suggesting that we might use the dipole approximation in Figure 4 as a matched filter to automate the process of locating and suppressing cultural anomalies. We implemented a "deculturing" scheme based on this idea by forming the crosscorrelation between the dipole of Figure 4 and each flight line profile. A crosscorrelation peak identifies the location of a presumed cultural anomaly. The anomaly is then reduced to approximately zero amplitude via multiplication by an appropriate window function. We used a $[1$ Hanning] window (width $=940 \mathrm{~m}$ ) which preserved the mean value as determined from the first and last $10 \%$ of the window width.

Starting with the largest crosscorrelation peak, we locate and suppress successively smaller anomalies, stopping when the cross correlation falls below a specified percentage (threshold) of the largest value within the profile. Figure 5a shows one flight line profile and the associated crosscorrelation. The remainder of Figure 5 shows "decultured" profiles for crosscorrelation thresholds of 90,50, 25, and 


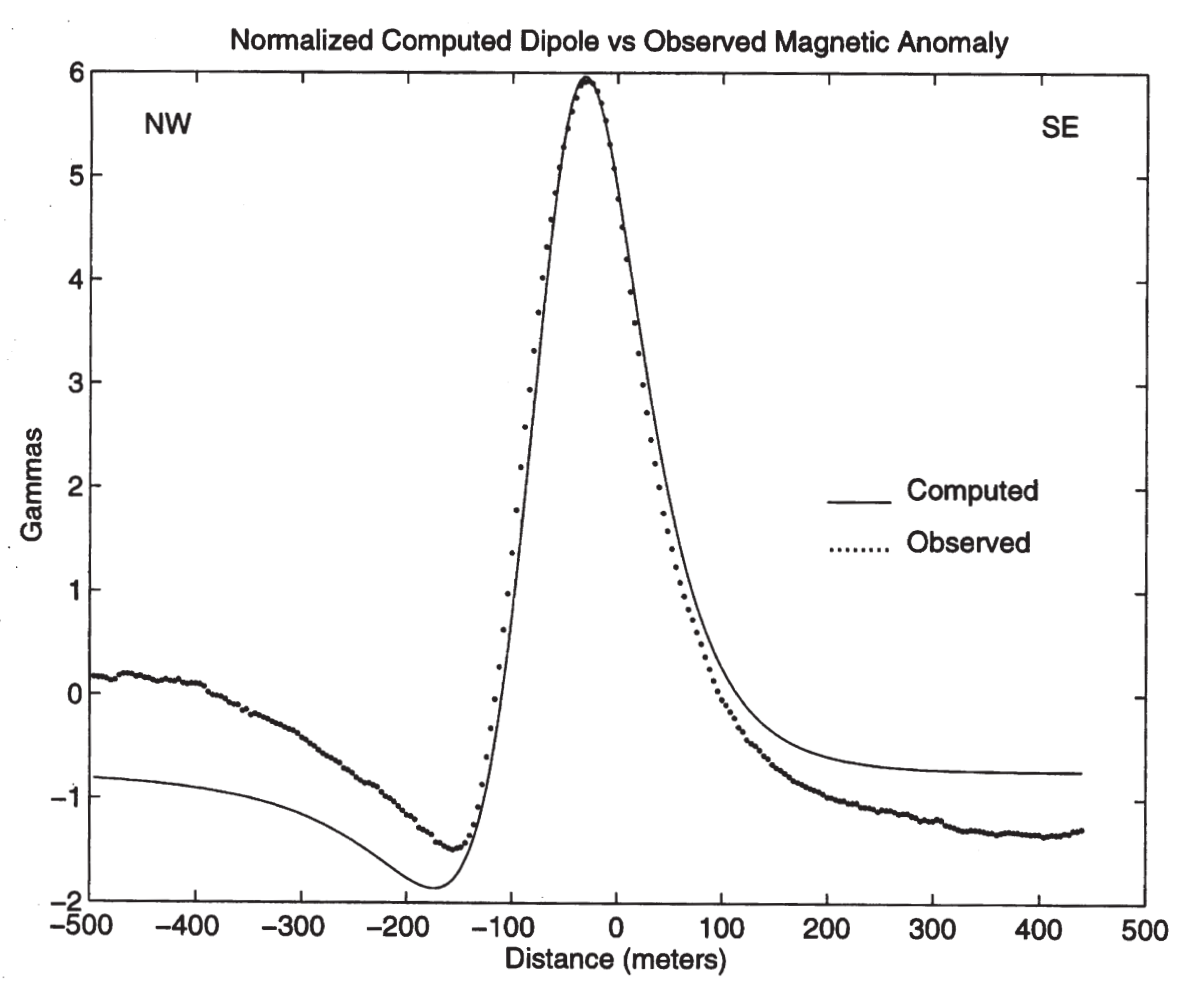

Figure 4. Observed magnetic anomaly over Khouri 1, and the modeled dipole anomaly. Dipole parameters are: elevation $=120 \mathrm{~m}$; offset from flight line $=5$ m; susceptibility $=300000$; field inclination $=60^{\circ}$; declination $=10^{\circ}$; flight path azimuth (relative to true north) $=320^{\circ}$.

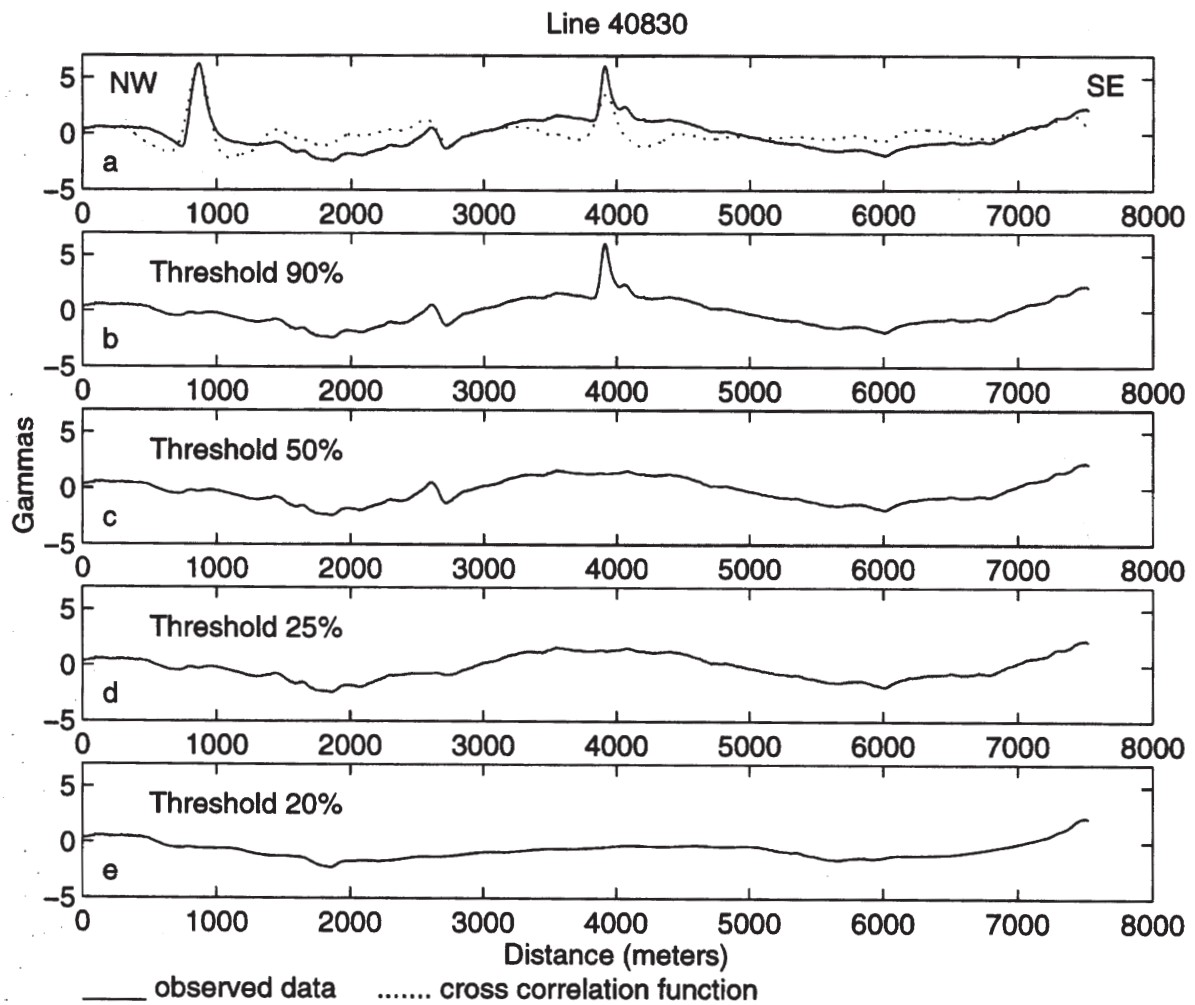

Figure 5. 5a shows the original flight profile and the crosscorrelation with the dipole of Figure 4. Peaks identify the location of cultural features having a similar dipole shape. The effect of changing the suppression threshold is illustrated in b-e. Suppressing cultural anomalies exceeding a threshold of $25 \%$ of the largest crosscorrelation peak appears suitable for these data.
$20 \%$. The selection of a threshold depends on the assumed character of the "signal" (sedimentary anomalies). At a threshold of $25 \%$, features that are most likely to be cultural have been suppressed, while a lower threshold $(20 \%)$ begins to affect anomalies that may be from sedimentary sources.

Using a 25\% threshold, we decultured portions of the survey and then applied a high pass zerophase shift filter; the results are shown in Figure 6. The deculturing scheme has greatly improved the ability to observe very low level anomalies, and appears to have removed most obvious cultural features. Of course, we may have also removed some "signal" because, as shown below, some sedimentary anomalies could have fairly large amplitudes. We note that one apparently cultural feature, indicated by the $\mathrm{C}$ on line 40770 (Figure 3) remains in Figure 6. C appears to have a slightly smaller wavelength, and a more symmetric shape than the dipole of Figure 4, and does not generate a large enough crosscorrelation peak to exceed the $25 \%$ threshold. C is located approximately where the highest magnetic susceptibility samples were gathered, as discussed below.

Sedimentary sources of magnetic anomalies. Magnetic anomaly sources within the shallow sedimentary section are almost certainly due to lateral variations in concentrations of magnetic minerals. We surveyed magnetic susceptibilities by gathering samples along public-access paved and unpaved roads. Samples were usually sandy and unconsolidated, although at a few locations more lithified or clay-rich material was encountered. Sample color varied considerably, from very red to gray and green-yellow, but there turned out to be no clear correlation between color and magnetic susceptibility. Because there are few road cuts in this area of gentle topography, most samples were gathered using a shovel to dig a hole near the fence line to a depth of $15-40 \mathrm{~cm}$. With a plastic spoon, we scraped off material near the hole bottom, then gathered a sample, and sealed it in a plastic bag. Susceptibility values were obtained the next day in the laboratory using a 

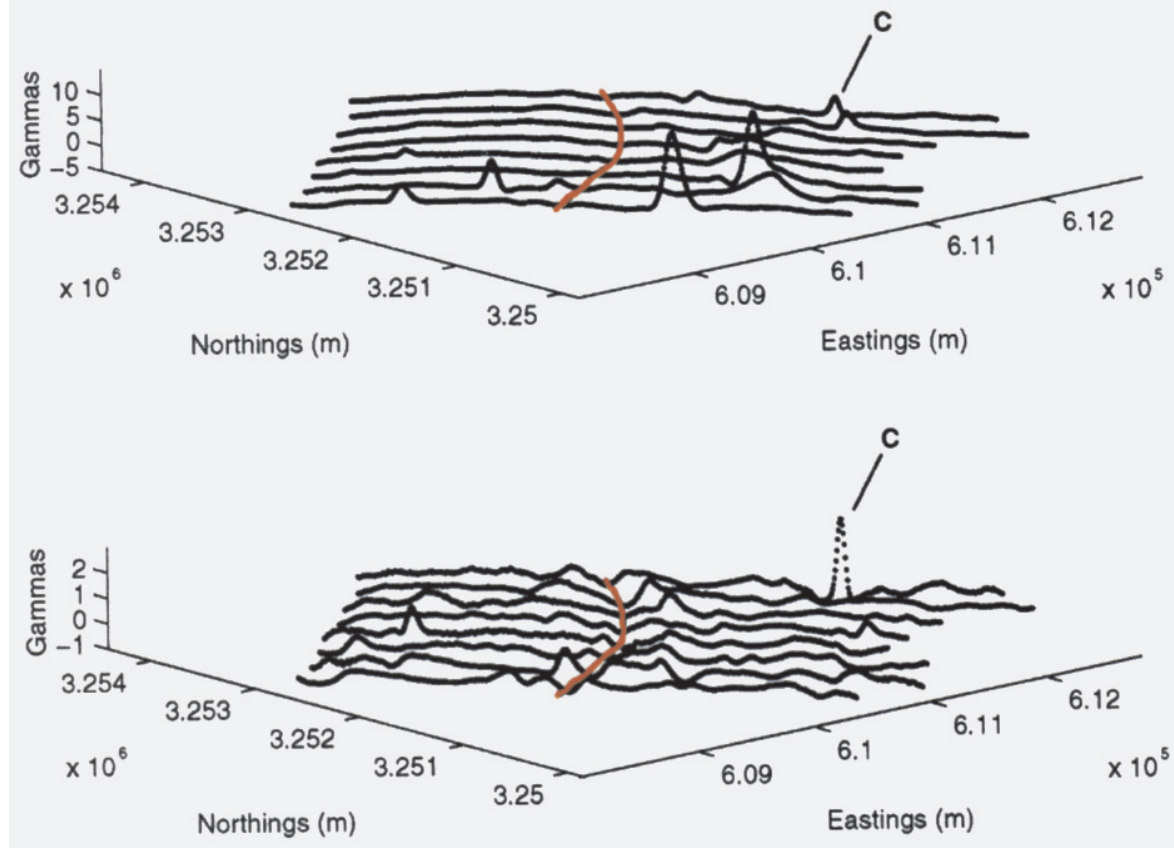

$\overline{0}$ meters 1000

Figure 6. Comparison of flight lines from Figure 3, before and after deculturing using a $25 \%$ threshold as in Figure 5 . Deculturing is followed by high pass filtering with a zero phase filter. Note the difference in amplitude scale. The low level anomaly is highlighted in red. See text for a discussion of anomaly $\mathrm{C}$.

Bartington MS-2 susceptibility meter. In a reconnaissance survey along Wilson County road 1681 (southern sample points in Figure 2), samples were taken roughly every $800 \mathrm{~m}$. Susceptibility ranged from near 1 to about 40, (in cgs units $\times 10^{* *} 6$ ). About $90 \%$ of the susceptibility values fell within the range 3-30, with a mean value of about 15 . For comparison, susceptibilities observed in natural sediments may be as large as several thousand, while sediments with susceptibilities near 1, at the lower limit of detectability with the Bartington meter, are considered nonmagnetic. A more detailed survey was conducted along unpaved county road 134 (northern sample points in Figure 2, also called Dewberry Road) which is located directly beneath some of the survey lines in Figures 3 and 6 . Samples were collected about every $150 \mathrm{~m}$. Measured susceptibilities towards the west end of the road were similar to those found in the reconnaissance survey, but higher values in the range of 100300 , were obtained near the east end, with order of magnitude changes over distances as small as a few hundred meters. The susceptibility profile is presented in Figure 7.

Mineralogy of surface samples. Powder X Ray Diffraction (XRD) was attempted, but quartz and microcline, the two major clastic sediment components, dominated the XRD patterns. Thus, XRD was not useful for identifying other constituents, such as magnetic minerals, which are present only in trace amounts. Isothermal remagnetization was used to identify the predominant magnetic minerals. This technique determines the level of applied field which produces saturation, i.e., no increased remanence with an increase in applied field, which is diagnostic of the principal magnetic minerals. Samples were mixed with sodium silicate, molded into $2 \times 2 \times 1 \mathrm{~cm}$ "bricks" and allowed to harden.

Progressively stronger magnetic fields were applied at room temperature in about 20 steps up to a maximum of 10 kilo-Oersteds (kOe). After each step, remanent magnetism was measured. We used a spinner magnetometer because, for most samples, remanent magnetism was too strong for our cryogenic magnetometer. All but one of the tested samples saturated with an applied field near 1.5-2 kOe, indicating a predominance of magnetite. One sample acquired most of its remanence near 1.5-2 kOe, but showed a slow increase in remanence for larger applied fields, suggesting an additional contribution from hematite.

Magnetic anomaly calculations. GMSYS software was used to predict total field anomalies at the $\sim 100 \mathrm{~m}$ aircraft altitude using a block two-dimensional magnetization model taken from measured susceptibilities along county road 134, which trends east-west, at an angle of about $45^{\circ}$ relative to the flight profiles. The model susceptibilities were invariant perpendicular to the road, and constant to a depth of $100 \mathrm{~m}$. The result, in Figure 7, shows that anomalies of a several $\gamma$, to almost $10 \gamma$ are expected. A portion of line 40770 is displayed alongside the predicted anomaly profile.

Anomaly $\mathrm{C}$ is located approximately where flight line 40770 intersects the east end of the susceptibility sample profile, which is also near the location of the peak predicted anomaly. The source of anomaly $C$ therefore could be high susceptibility sediments, rather than a cultural source.

Discussion. Based on observed amplitudes $(0.5-1 \gamma)$, spatial scales, strike orientation, correlation across several flight lines, and observed surface susceptibility variations, the low level anomalies identified in Figures 2, 3, and 6 appear to be of natural origin, arising from magnetite variations in near-surface sediments. We favor the hypothesis that near-surface magnetite variations are original depositional features, with no causal relationship to petroleum occurrence, but other explanations are not excluded by our data. Bacterial enrichment of near-surface magnetite in the presence of hydrocarbons seems unlikely, given the lack of reported oil seeps, but additional mineralogical studies would be required to determine whether postdepositional chemical mechanisms have also affected magnetic mineral concentrations.

Modern aeromagnetic total field data are an order of magnitude bet- 

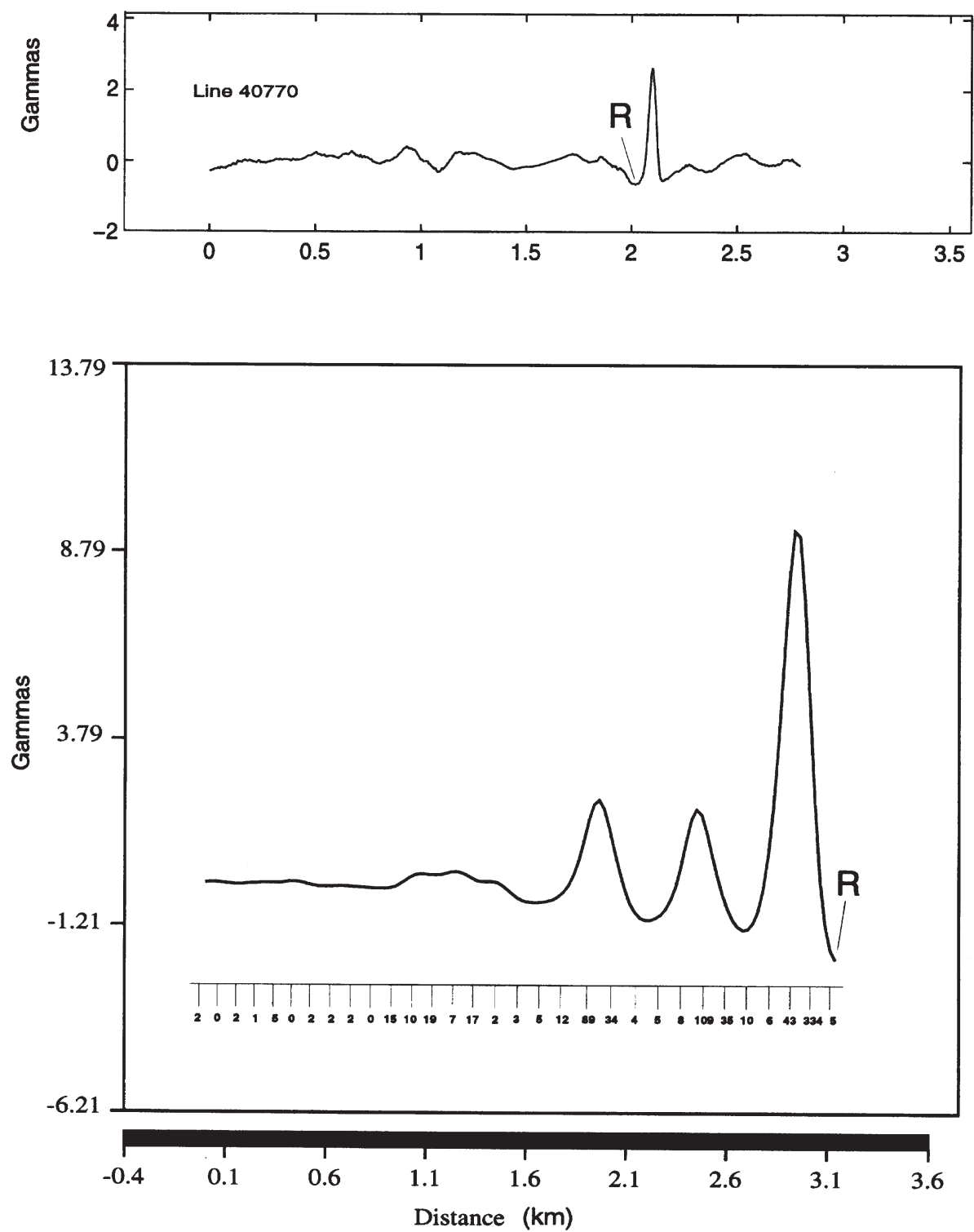

Figure 7. High resolution magnetic susceptibility profile, and predicted anomaly at $100 \mathrm{~m}$ altitude, assuming a block model with susceptibility variations obtained from surface samples along County Road 134. Line 40770 is displayed with the predicted anomaly. The two profiles are aligned at their intersection point, which is approximately the east end of County Road 134 marked by " $R$ " where the highest susceptibility samples were obtained.

ter than a decade ago, permitting detection of anomalies with spatial scales of tens of meters and amplitudes below $1 \gamma$. Unfortunately, in exploration applications inhabited areas, there is inevitable cultural contamination. When cultural anomaly amplitudes are considerably larger than those from natural sources, and have a characteristic shape, such as that of a dipole, an anomaly suppression scheme appears useful, although it needs to be applied cautiously. For example, by selecting a lower threshold, anomaly $C$, which may have a nat- ing data on water tanks and other metal structures. Separation of cultural from natural-source anomalies can also be assisted by methods familiar in seismic studies, including a good understanding of the geological setting, knowledge of physical property (magnetic susceptibility) variations, and suitable data display techniques.

Suggestions for further reading. Good case studies of magnetic anomalies in areas of petroleum production are: Sources of aeromagnetic anomalies over Cement Oil Field (Oklahoma), Simpson Oil Field (Alaska), and WyomingIdaho-Utah Thrust Belt" by Reynolds et al. (GEOPHYSICS, 1991). Articles describing physical mechanisms for magnetic anomaly production related to hydrocarbons include "Chemical and microbial processes causing anomalous magnetization in environments affected by hydrocarbon seepage" by Machel and Burton (GEOPHYSICS, 1991); "Evidence for a relationship between hydrocarbons and authigenic magnetite" by Elmore et al. (Nature, 1987); "Relations among hydrocarbon reservoirs, epigenetic sulfidization and rock magnetization, examples from the South Texas coastal plain" by Goldhaber and Reynolds (GEOPHYSICS, 1991).

Examples of non-petroleum-related anomalies in sedimentary environments are given by Gay and Hawley in "Syngenetic magnetic anomaly sources: Three examples" (GEOPHYSICS, 1991). E

Acknowledgements: This study was supported by the State of Texas under the Advanced Technology Program Grant No. 372 of the Texas Higher Education Coordinating Board. Additional support came from the University of Texas Institute for Geophysics, World Geosciences, and the Geology Foundation of the University of Texas. BGM Airborne Surveys and Texas Instruments provided sup port through cost sharing. Geosoft, Earth Resources Mapping, GMSYS, and Geographix donated computer software. Tobin surveys, Graphics Information Technology, Desing Technology, Petroleum Information, and GEOMAP provided cultural geograhic information data. We greatly appreciate the assistance of Wulf Gose with magnetic mea surements and that of Fangqiong $L u$ with $X R D$.

Corresponding author: Clark Wilson, fax 2023582770; emailcwilson@hq.nasa.gov. 